\title{
Diffuse miliary hemangiomatosis with hepatic involvement in an infant in Dakar
}

\section{Khadim Diop', Maodo Ndiaye', Mame Téné Ndiaye ${ }^{2}$, Coumba Ndiaye', Boubacar Ahy Diatta1', Saer Diadier', Niare Ndour'1, Aminata Deh¹, Moussa Diallo', Suzanne Oumou Niang ${ }^{1}$, Kane Assane ${ }^{2}$}

${ }^{1}$ Service de Dermatologie, Hôpital Aristide Ledantec, Dakar, Senegal, ${ }^{2}$ Service de Dermatologie, Hôpital d'enfants Albert Royer, Dakar, Senegal

Corresponding author: Khadim Diop, MD, E-mail: bambadiop100391@gmail.com

\begin{abstract}
ABSRACT
We report the case of a 03 -month-old female infant with no specific history, who was admitted in the Pediatric Department for an abdominal tumor that has evolved for 01 month. The physical examination noticed a large hepatomegaly and multiple raspberry-like papulo-nodules located on the trunk, scalp and limbs. The alpha-fetoprotein level was $842 \mathrm{ng} / \mathrm{ml}$ (42 times normal). The abdominal ultrasound and the CT scan made it possible to retain the diagnosis of diffuse miliary hemangiomatosis with liver damage. A favorable evolution was noted with propanolol and corticosteroids after a follow-up of 04 months.
\end{abstract}

Key words: Miliary hemangiomatosis; Liver; Alpha-fetoprotein; Senegal

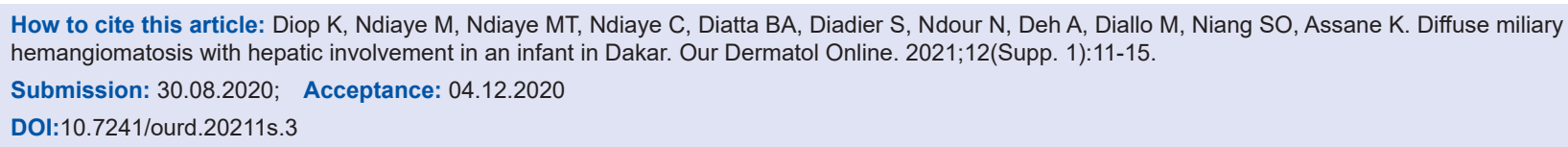




\section{Une hémangiomatose miliaire diffuse avec atteinte hépatique chez un nourrisson à Dakar}

\section{Khadim Diop', Maodo Ndiaye', Mame Téné Ndiaye ${ }^{2}$, Coumba Ndiaye', Boubacar Ahy Diatta', Saer Diadier', Niare Ndour'1, Aminata Deh¹, Moussa Diallo', Suzanne Oumou Niang', Kane Assane ${ }^{2}$}

${ }^{1}$ Service de Dermatologie, Hôpital Aristide Ledantec, Dakar, Senegal, ${ }^{2}$ Service de Dermatologie, Hôpital d'enfants Albert Royer, Dakar, Senegal

Corresponding author: Khadim Diop, MD, E-mail: bambadiop100391@gmail.com

\section{RÉSUMÉ}

Nous rapportons l'observation d'un nourrisson de 03 mois, de sexe féminin, sans antécédents particuliers, hospitalisé au service de Pédiatrie pour une tumeur abdominale évoluant depuis 01 mois. L'examen physique notait une volumineuse hépatomégalie tumorale et de multiples papulo-nodules framboisés localisés sur le tronc, le cuir chevelu et les membres. Le taux d'alpha foeto-protéine était à $842 \mathrm{ng} / \mathrm{ml}$ (42 fois la normale). Léchographie abdominale complétée par le scanner ont permis de retenir le diagnostic d'hémangiomatose miliaire diffuse avec atteinte hépatique. Lévolution était favorable sous propanolol et corticoïdes après un recul de 04 mois.

Mots-clés : Hémangiomatose miliaire; Hépatique; Alpha fotoprotéine; Sénégal 


\section{INTRODUCTION}

L'hémangiomatose miliaire diffuse correspond à une efflorescence d'hémangiomes cutanés infantiles dont le nombre est supérieur ou égal à cinq associés à une atteinte viscérale, le plus souvent hépatique [1-14]. La gravité est liée surtout au risque d'insuffisance cardiaque congestive [2]. C'est une affection rare, représentant 2,5 à 3 $\%$ des cas d'hémangiomes cutanés [1-3]. Nous rapportons une observation d'hémangiomatose miliaire diffuse avec atteinte hépatique chez un nourrisson au Sénégal.

\section{OBSERVATION}

Il s'agissait d'un nourrisson de 03 mois, de sexe féminin, hospitalisé au service de Pédiatrie de l'hôpital Aristide Le Dantec pour une tumeur abdominale d'évolution progressive depuis un mois. Il était né d'un accouchement par voie basse à 38 semaines d'aménorrhée d'une grossesse mono-fœetale bien suivie avec un score d'Apgar à la naissance à 10 et le poids de naissance à 2900 grammes. La mère était âgée de 20 ans, troisième geste, sans antécédents particuliers.

A l'examen général, il existait une volumineuse tuméfaction abdominale avec une peau tendue luisante et de multiples circulations veineuses collatérales (Figure 1). Le nourrisson était agité avec des pleurs incessants, une fréquence respiratoire à 42 cycles/ minute et une fréquence cardiaque à 160 battements/ minute. La saturation était à $95 \%$ et la température à $37,2^{\circ} \mathrm{C}$. Lexamen dermatologique retrouvait des papulo-nodules d'aspect framboisé au nombre de 9, localisés sur le cuir chevelu, au niveau temporal, le dos, le décolleté et les membres supérieurs, évoquant des

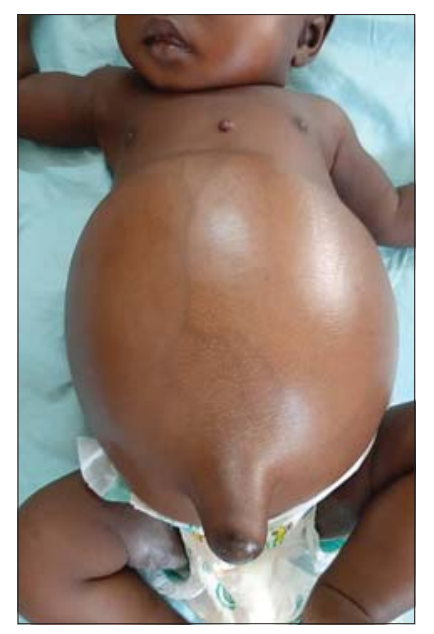

Figure 1: Hépatomégalie tumorale avec de multiples circulations veineuses collatérales épigastrique. hémangiomes tubéreux (Figs. 1 - 3). Lexamen digestif objectivait une volumineuse hépatomégalie d'allure tumorale. Lexamen cardio-pulmonaire ne retrouvait pas d'anomalies en dehors d'une tachycardie et le reste de l'examen clinique était normal. Léchographie hépatique montrait une hépatomégalie avec de multiples formations hypoéchogénes diffuses sur tout le parenchyme hépatique et complétée par la tomodensitométrie abdominale qui objectivait un volumineux foie, dysmorphique, hétérogéne, siège de plusieurs formations hypodenses, diffuses sur tout le parenchyme et rehaussées par le produit de contraste. Lélectrocardiogramme montrait une tachycardie sinusale régulière. La radiographie du thorax était normale. L'hémogramme montrait une anémie hypochrome microcytaire à $5,5 \mathrm{~g} / \mathrm{dl}$, les leucocytes étaient à $8400 / \mathrm{mm}^{3}$ et les plaquettes à $456000 / \mathrm{mm}^{3}$. Les ASAT étaient à 50 UI et les ALAT à 26 UI. Le taux d'alpha foeto-protéine était à $842 \mathrm{ng} / \mathrm{ml}$ (42 fois la normale). La glycémie à jeûne, la fonction rénale, l'ionogramme sanguin et le taux de LDH étaient normaux. Le diagnostic d'une hémangiomatose miliaire diffuse avec atteinte hépatique était retenue devant la présence d'hémangiomes cutanés multiples et l'aspect radiographique de l'atteinte hépatique. Un traitement par voie orale associant la bétaméthasone 10 gouttes/kg à dose dégressive et du propanolol 2/ $\mathrm{mg} / \mathrm{kg}$ était administré. Lévolution était favorable, après un recul de 04 mois, marquée par une régression de l'hépatomégalie, un début d'affaissement des hémangiomes et une tendance à la normalisation du taux d'alpha foto-protéine à deux fois la normale (Fig. 4).

\section{DISCUSSION}

L'hémangiomatose miliaire est une affection rare, représentant 2,5 à $3 \%$ des cas d'hémangiomes infantiles cutanés [1-3]. La présence d'hémangiomes multiples



Figure 2: Multiples hémagiomes tubéreux sur le dos. 
sur la peau est depuis longtemps reconnue comme marqueurs des hémangiomes hépatiques et à rechercher systématiquement par une échographie hépatique [4].

Notre observation est particulière par sa présentation clinique une atteinte hépatique tumorale, une forte élévation du taux d'alpha foeto-protéine, le retentissement cardiaque et de par son profil évolutif. En effet, l'hépatomégalie d'allure tumorale avec une élévation importante du taux d'alpha foeto-protéine faisait évoquer en premier lieu un hépatoblastome et un carcinome hépatocellulaire. Cependant la présence d'hémangiomes cutanés multiples et la mise évidence d'une hépatomégalie avec de multiples formations hypoéchogènes homogénes à l'échographie abdominale ont permis de retenir le diagnostic d'une hémangiomatose miliaire diffuse malgré la forte élévation du taux d'alpha protéine [5,6]. En effet, l'échographie hépatique avec étude doppler constitue l'examen de première intention pour la mise en évidence des hémangiomes hépatiques, qui est habituellement sphérique, hypoéchogène et homogéne [1,5-12]. Le

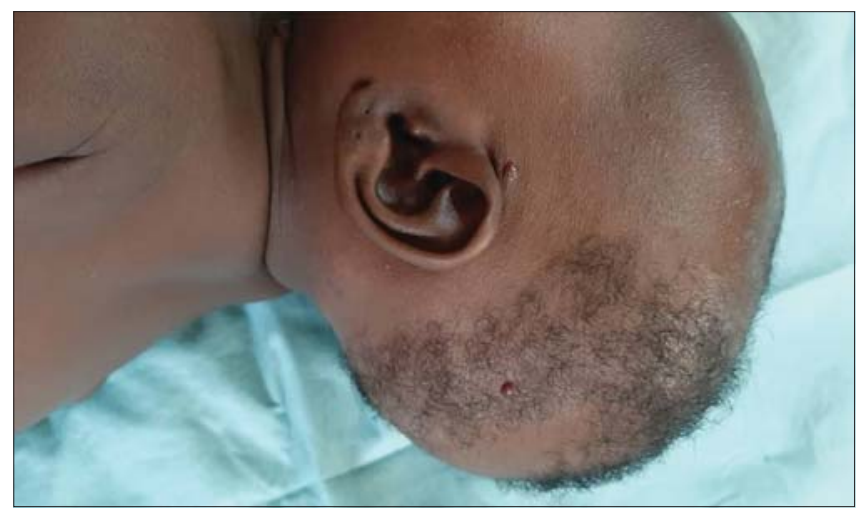

Figure 3: Hémangiomes tubéreux sur la région occipital et temporal.

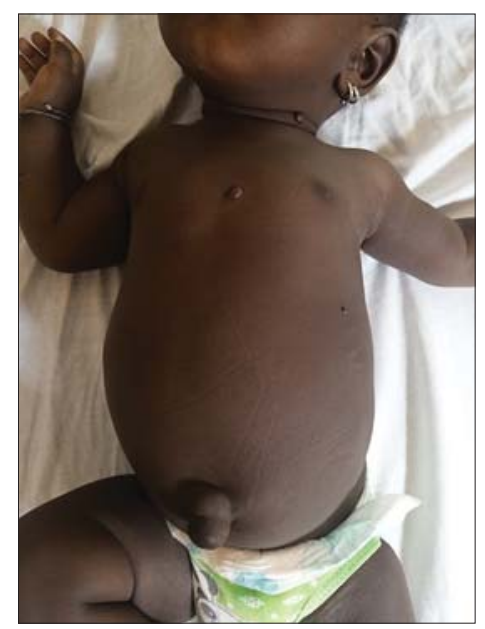

Figure 4: Régression de l'hépatomégalie tumorale et début d'affaissement des hémangiomes cutanés après traitement. scanner abdominal ou mieux l'imagerie par résonnance magnétique sont indiqués dans de rares cas, lorsque l'aspect échographique est atypique [12].

Une autre particularité de notre observation est l'élévation importante du taux d'alpha foeto-protéine chez notre malade. En effet, l'élévation de cette protéine sérique n'est pas classique au cours de l'hémangiomatose miliaire diffuse avec atteinte hépatique. Seuls, quelques auteurs ont rapporté une élévation importante du taux d'alpha foetoprotéine associée à des hémangiomes hépatiques [8-11]. En Inde, In Sook Seo et al, avaient rapporté une série de 05 cas d'hémangiomes hépatiques associés à une élévation importante du taux d'alpha foto-protéine [8]. Herman, Siegal et al, Lehrenbecher et al, Urbach et al, avaient, eux aussi, rapportés des cas isolés [9-11]. Laugmentation des taux élevés d'alpha foto-protéine sérique au cours des hémangiomes hépatiques infantile n'est pas documentée, à ce jour [11]. Elle serait dûe, d'une part, à une production importante de cette protéine sérique au cours de l'embryogenèse. Celleci s'effectue au début par la vésicule ombilicale puis par les hépatocytes et la muqueuse gatro-intestinale. Elle atteint sa concentration maximale entre 12 et 14 semaines de gestation, diminue ensuite et atteint sa concentration normale à l'âge de 08 à 12 mois. D'autre part, on suppose qu'elle serait secrétée par les cellules hémangioendothéliales, mais cela n’a pas été démontré par l'immunohistochimie. Lautre hypothèse serait une production par le tissu hépatique normal en réponse aux tumeurs [11].

La gravité de l'hémangiomatose miliaire diffuse avec hépatomégalie est liée, surtout au risque d'insuffisance cardiaque à haut débit par la formation de shunt artérioveineux. D'autres complications peuvent survenir notamment les compressions des organes de voisinage par effet de masse, une anémie et une hypothyroïdie par sécrétion inappropriée d'iodothyronine déiodinase 3 [1]. Chez notre malade, les signes d'intolérance étaient la dyspnée, la tachycardie, la présence de multiples circulations veineuses collatérales abdominale et l'anémie.

L'efficacité du traitement médical seul dans l'hémangiomatose miliaire diffuse avec atteinte hépatique a été souligné par de nombreux auteurs [12,13]. Plusieurs médicaments ont été proposés seul ou en association : le propanolol, les corticoïdes systémiques, la vincristine et l'interféron [1-12]. L'étude de Mhanna et al, a montré une efficacité spectaculaire du propanolol sur une série d'hémangiomatose cutanée et hépatique [12]. Chez 
notre malade, du fait de l'effet de masse important de l'hépatomégalie avec signes de mauvaise intolérance, nous avons associé la bétaméthasone à 10 gouttes/ $\mathrm{kg}$ à dose dégressive et le propanolol à $2 \mathrm{mg} / \mathrm{kg}$. Ceci a permis, après un recul de 4 mois, une régression de l'hépatomégalie, un début d'affaissement des hémangiomes cutanés et une tendance à la normalisation du taux d'alpha foeto-protéine, qui est passé de 42 fois la normale à 2 fois la normale.

\section{CONCLUSION}

Notre observation est particulière par sa présentation clinique à type d'atteinte hépatique tumorale, une forte élévation du taux d'alpha foeto-protéine, le retentissement cardiaque et une bonne évolution sous corticoïde et bétabloquant.

\section{Consent}

The examination of the patient was conducted according to the principles of the Declaration of Helsinki.

The authors certify that they have obtained all appropriate patient consent forms, in which the patients gave their consent for images and other clinical information to be included in the journal. The patients understand that their names and initials will not be published and due effort will be made to conceal their identity, but that anonymity cannot be guaranteed.

\section{RÉFÉRENCES}

1. Lacoste A, Léauté-Labrèze C, Hémangiomatose miliaire. Ann Dermatol Venereol. 2007;134:694-8.
2. Stanley P, Geer D, Miller J, Gilsanz V, Landing B, Ines M. et al. Infantile hepatic hemangiomas. Cancer. 1989;64:936-49.

3. Haggstrom AN, Drolet BA, Baselga E, Chamlin SL, Garzon MC, Horii KA, et al, Prospective study of infantile hemangiomas: clinical characteristics predicting complications and treatment. Pediatrics. 2006;118:882-7.

4. Dickie B, Dasgupta R, Nair R, Alonso MH, Ryckman FC, Tiao GM, et al. Spectrum of hepatic hemangiomas: management and outcome. J Pediatr Surg. 2009;44:125-33.

5. Gnarra M, Behr G, Kitajewski A, Wu JK, Anupindi SA, Shawber CJ, et al. History of the infantile hepatic hemangioma: From imaging to generating a differential diagnosis. World J Clin Pediatr. 2016;5:273-80.

6. Meyers RL. Tumors of the liver in children. Surg Oncol. 2007;16:195-203.

7. Christison-Lagay ER, Burrows PE, Alomari A, Dubois J, Kozakewich HP, Lane TS, et al, Hepatic hemangiomas: subtype classification and development of a clinical practice algorithm and registry. J Pediatr Surg, 2007;42:627.

8. Seo IS, Min KW, Mirkin LD. Hepatic hemangioendothelioma of infancy associated with elevated alpha fetoprotein and catecholaminine by-products. Pediatr Pathol. 1988;8:625-31.

9. Urbach AH, Zitelli BJ, Blatt J, Gartner JC, Malatack JJ. Elevated alpha fetoprotein in a neonate with a benign hemangioendothelioma of the liver. Pediatrics. 1987;80:596-97.

10. Herman TE, Siegel MJ. Solitary hepatic hemangioendothelioma with extensive cystic necrosis and markedly elevated alpha fetoprotein. J Perinatal. 2001;21:568-70.

11. Sari N, Yalçin B, Akyüz C, Haliloglu M, Büyükpamukçu M. Infantile hepatic hemangioendothelioma with elevated serum alpha-fetoprotein. Pediatr Hematol Oncol. 2006;23:639-47.

12. Mhanna A, Franklin WH, Mancini AJ. Hepatic infantile hemangiomas treated with oral propranolol-a case series. Pediatr Dermatol. 2011;28:39-45.

13. Mazereeuw-Hautier J, Hoeger PH, Benlahrech S, Ammour A, Broue P, Vial J, et al. Efficacy of propranolol in hepatic infantile hemangiomas with diffuse neonatal hemangiomatosis. J Pediatr. 2010;157:340-2.

14. Mahamadou G, Saka B, Mouhari-Toure A, Tchaou M, Pessinaba S, Gnassingbé W, et al. [Benign neonatal hemangiomatosis: An observation in a child in Togo]. Ann Dermatol Venereol. 2017;144:655-6. 